\title{
Is Overnight Fasting before Surgery Too Much or Not Enough? How Basic Aging Research Can Guide Preoperative Nutritional Recommendations to Improve Surgical Outcomes: A Mini-Review
}

\author{
Alban Longchamp ${ }^{a, b} \quad$ Eylul Harputlugil ${ }^{a}$ Jean-Marc Corpataux ${ }^{c}$ \\ C. Keith Ozaki ${ }^{b}$ James R. Mitchell ${ }^{a}$ \\ ${ }^{a}$ Department of Genetics and Complex Diseases, Harvard T.H. Chan School of Public Health, and ${ }^{b}$ Department \\ of Surgery and the Heart and Vascular Center, Brigham and Women's Hospital and Harvard Medical School, \\ Boston, MA, USA; ' Department of Vascular Surgery, Centre Hospitalier Universitaire Vaudois, and Lausanne \\ University, Lausanne, Switzerland
}

\section{Key Words}

Dietary restriction - Surgery - Surgical stress - Nutrition .

Hydrogen sulfide $\cdot$ Amino acid sensing · Ischemia .

Longevity . Enhanced recovery after surgery

\begin{abstract}
Dietary restriction (DR) is best known for extending lifespan in experimental model organisms, but also increases resistance to a variety of clinically relevant stressors, including those associated with surgery. Extended periods of DR, lasting months to years, are required for optimal longevity benefits in rodents, but short-term dietary preconditioning (less than 1 week) remarkably protects from acute injury. Here, we discuss recent advances in our understanding of the mechanistic basis of short-term DR and fasting in the context of surgical stress resistance, including upstream amino acid sensing by the GCN2 and mTORC1 pathways, and downstream effector mechanisms including increased insulin-dependent prosurvival signaling and elevated endogenous hydrogen sulfide production. We also review the current trend in preoperative nutrition away from preoperative fasting and towards carbohydrate loading. Finally, we discuss the
\end{abstract}

\section{KARGER}

(c) 2017 S. Karger AG, Basel

E-Mail karger@karger.com

www.karger.com/ger rationale for the nonmutually exclusive use of brief DR or pharmacological DR mimetics to precondition against the stress and potential complications of surgery.

(c) 2017 S. Karger AG, Basel

\section{Introduction}

In 1935, Clive McCay reported that dietary restriction (DR), or reduced food intake without malnutrition, extends lifespan of laboratory rats $[1,2]$. Since that time, longevity extension by DR has been demonstrated in numerous experimental organisms from yeast to non-human primates [1]. DR is thought to extend longevity by reducing the rate of aging, making it a key tool in aging research. However, since maximal lifespan extension requires long-term adhesion, antigeronic clinical applications have never been considered feasible due to the obvious difficulties associated with long-term voluntary food restriction. Indeed, while prospective clinical trials show that DR can reduce risk factors for diabetes, cardiovascular disease, and cancer, they also highlight problems with long-term compliance $[3,4]$. 
Fortunately, DR confers other important benefits that do not require long periods of food restriction, including increased resistance to multiple forms of acute stress. One of the biggest planned stressors many people will face in their life is that of major elective surgery, which carries inherent risks of complications, such as perioperative stroke and myocardial infarction (MI).

A novel concept in surgical risk mitigation emerging from basic research on DR and aging is dietary preconditioning, or short-term DR lasting one week or less prior to surgery [5]. In rodent models of surgical stress ranging from ischemia reperfusion injury (IRI) to vascular restenosis (intimal hyperplasia), short-term DR or fasting before surgery, followed by a return to normal food intake after surgery, leads to improved outcomes [6-10].

Here, we will provide an update on recent advances in our understanding of the nutritional basis, nutrient sensing mechanisms, and downstream effectors of protection by short-term DR and fasting in preclinical models of surgical stress. Finally, we will discuss the translational potential of DR and DR mimetics before surgery in relation to current practices.

\section{The Surgical Stress Response and Associated Complications}

Surgery is an invasive medical intervention involving an incision, with major surgery typically describing procedures in which a body cavity is entered. Like many types of acute injury, surgery perturbs metabolic and immune homeostasis through effects on afferent (autonomic and sympathetic) nerve input from the area of trauma leading to local and systemic catecholamine release, increased levels of proinflammatory acute phase reactants, metabolic adaptations including glycogen mobilization, and vascular changes including vasoconstriction and increased heart rate. Parallel activation of the hypothalamic-anterior pituitary-adrenomedullary axis promotes release of cortisol from the adrenal cortex, resulting in a partially counterbalancing response characterized by protein and fat mobilization, immunosuppression, and dampening of the action of anabolic hormones such as insulin and testosterone [11]. Together, these effects result in a transient increase in metabolic rate that scales in amplitude and duration with the severity of the insult (Fig. 1a).

From an evolutionary standpoint, the selective advantage conferred by these coordinated stress responses likely involves staunching hemorrhage and redirecting stored energy to immune function and tissue repair to promote

Is Overnight Fasting before Surgery Too Much or Not Enough? survival following acute injury. However, in any given surgical patient, such vasoconstrictive, prothrombotic and proinflammatory responses can also precipitate ischemia (inadequate blood supply), which underlies a range of surgical complications such as renal dysfunction, myocardial ischemia or stroke.

Risk assessments prior to surgery focus on the potential for a perioperative major adverse cardiovascular event (MACE), a composite of potential negative outcomes including MI and death (either MI-related or all-cause mortality), but also sometimes stroke or the need for urgent revascularization [12]. Estimations of risk, including the revised cardiac risk index (RCRI) or the American College of Surgeons National Surgical Quality Improvement Program risk (ACS-NSQIP), are based on patient characteristics such as age, renal function, metabolic status, cardiac and neurological history, and results of physical examination prior to surgery, but also on the type of surgery performed. For example, the majority of cardiac surgeries are performed on an ischemic, nonbeating heart achieved by cross-clamping the aorta (thus interrupting coronary artery perfusion), with systemic blood circulation achieved via extracorporeal circulation (ECC). In addition to IRI to the heart, ECC itself causes a systemic inflammatory response leading to activation of circulating leukocytes and a prothrombic state potentially contributing to cardiac dysfunction $[13,14]$.

Surgery also carries a risk of non-MACE complications specific to a particular procedure. For example, complications of open abdominal aortic aneurysm repair include limb, pelvic, mesenteric and spinal ischemia, while repair of aortic lesions involving the renal arteries carries increased risk of renal failure (due to inflow artery cross-clamping) which directly relates to the ischemic time [15]. Interestingly, while risk assessment is standard practice in deciding if the benefits of a given surgery outweigh its risks, interventional prophylactic approaches to reduce patient-based risk factors prior to surgery are not.

\section{Short-Term DR Preconditions against Surgical Stress in Rodent Models}

The term DR is used here to describe a variety of dietary regimens with overlapping functional benefits including extended longevity, improved metabolic fitness and increased stress resistance. While the nutritional basis of DR is described in more detail below, such regimens can generally be divided into two categories. The first involves calorie restriction, for example by daily restriction 


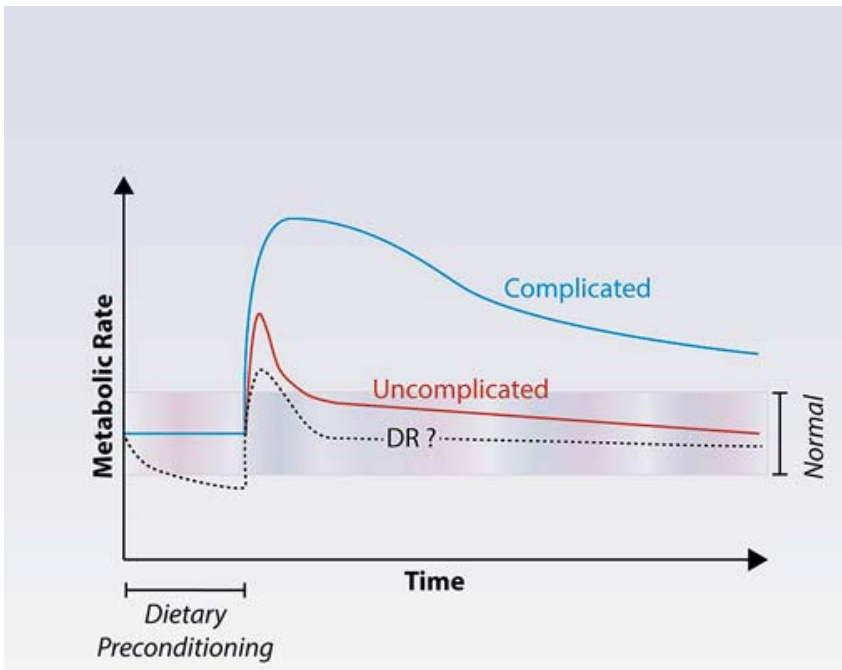

a

Fig. 1. Potential effects of DR on surgical stress resistance. a Model of the metabolic response to uncomplicated elective surgery vs. surgery in which complications such as MI, renal failure, or stroke occur. Also modeled is how DR affects metabolic rate prior to surgery as well as the metabolic response to surgical stress. b Cellular mechanisms of protection by dietary preconditioning. Dietary protein or essential amino acid restriction activates general control nonderepressible 2 (GCN2), AMP-activated protein kinase (AMPK), and sirtuin 1 (SIRT1) and represses mammalian target of rapamycin complex 1 (mTORC1) nutrient/energy sensing sig-

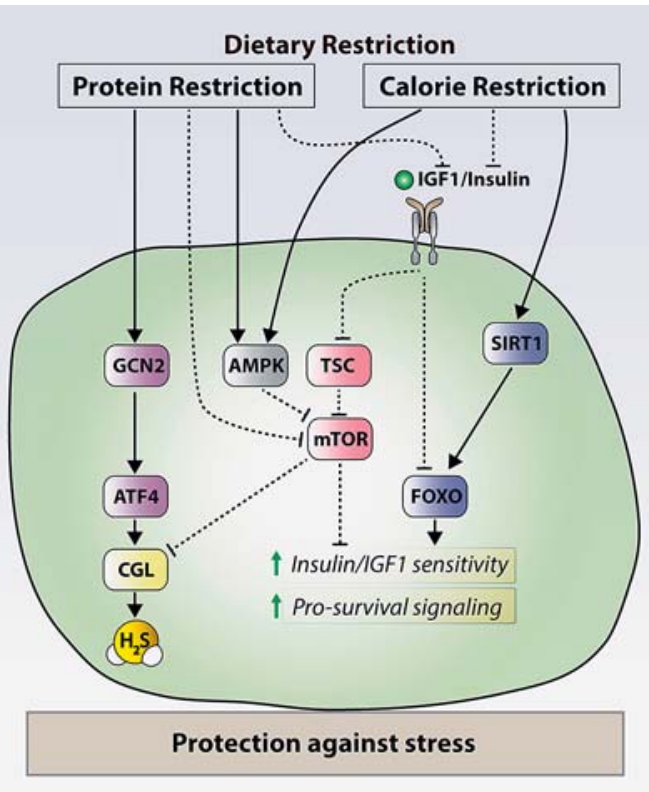

b nal transduction pathways, resulting in increased hydrogen sulfide $\left(\mathrm{H}_{2} \mathrm{~S}\right)$ production by the transsulfuration pathway enzyme cystathionine $\gamma$-lyase (CGL) and improved insulin/insulin-like growth factor 1 (IGF-1) receptor sensitivity. $\mathrm{H}_{2} \mathrm{~S}$ can protect against acute stress via numerous mechanisms including direct antioxidant action, while improved insulin/IGF-1 sensitivity allow for increased prosurvival signaling, contributing to overall stress resistance. ATF4, activating transcription factor 4; FOXO, forkhead box O1; ROS, reactive oxygen species. of food intake or periods of intermittent fasting. The second involves special diets reduced in specific nutrients such as protein, but fed on an ad libitum (unlimited) basis without enforced food/calorie restriction. Because DR and starvation are by definition mutually exclusive states, the duration of treatment is of critical importance, particularly in cases involving severe food restriction or diets lacking essential nutrients such as protein or individual essential amino acids.

As with any regimen necessitating dietary alterations, duration is also of critical importance when considering practical translation to the clinic. For the purposes of this review, we define short-term as a week or less, and longterm as greater than 1 week. Historically, DR benefits including life span extension, improved metabolic fitness and even decreased injury in preclinical models of surgical stress including IRI to heart or brain $[16,17]$ were originally described in association with long-term DR regimens lasting months to years and thus with little practical trans- lational potential. However, more recent experiments designed to test the timing of onset of DR benefits make it clear that rapid onset is an evolutionarily conserved property of DR-mediated stress resistance [18]. For example, 2-4 days of water-only fasting is sufficient for protection in models of organ injury including cold IRI, a model of organ storage prior to reattachment that is relevant to organ transplantation [19]. Similarly, 6-7 days of reduced total food or isolated protein intake protects against warm IRI to brain, liver or kidney $[8,10,20]$ relevant both to surgical procedures involving intentional interruption of blood flow, as well as unintended surgical complications including heart attack or stroke. Importantly, short-term protein restriction or fasting also protect from chronic re-occlusive vascular wall adaptations, such as intimal hyperplasia, which can result in recurrent end-organ ischemia, loss of limb, reduced brain function, or even death [7]. Because the etiology of hemodynamically-driven intimal hyperplasia is distinct from IRI, this finding demonstrates the ability of 
short-term DR to protect against multiple different kinds of surgery-associated acute and chronic complications.

While the protective effects of short periods of DR prior to surgery are becoming evident in preclinical models of surgical stress as described above, still very little is known about the ability of DR after injury (postconditioning) to confer any protection, with some studies showing no effect [8] and others some efficacy [21]. Additionally, the relevance of refeeding after surgery in mice that are restricted prior to surgery is unknown. Finally, whether the integrated neuroendocrine/metabolic response to surgery is dampened by dietary preconditioning, and to what extent this affects surgical outcome, remains to be examined (Fig. 1a).

\section{Nutritional Requirements for Dietary Preconditioning against Surgical Stress}

Classic DR regimens, often referred to as caloric restriction, involve restriction of total food intake resulting in proportional reduction of calories in the form of carbohydrates, fats, and protein. In experimental rodents, the amount of restriction leading to longevity benefits is typically in the range of $30-40 \%$, although benefits have been observed up to near the point of starvation around $60 \%$ restriction [22]. Logistically, there are a variety of ways to accomplish DR, ranging from daily allotment of a fixed amount of food to alternating periods of fasting and ad libitum food intake, with enforced periods of food restriction as the common denominator.

While protein is equal in caloric content to carbohydrate, restriction of protein (but not sugar or fat) can contribute to dietary preconditioning beyond its caloric value $[20,23]$. Although the extension of this finding to longevity has been questioned [24], preconditioning against surgical stress in preclinical models can be leveraged without enforced food restriction by using diets reduced in or lacking protein. This is an important difference when considering clinical translational potential due to the difficulties inherent in enforced restriction of food intake even for short periods of time.

Dietary protein is composed of approximately 20 amino acids present in different ratios depending on the source of protein. Of these, mammals can synthesize adequate amounts of approximately 10 "nonessential" amino acids, while the remaining "essential amino acids" must be obtained from the diet. In many animals including mammals, recent data indicate that the benefits of protein restriction are controlled by the restriction

Is Overnight Fasting before Surgery Too Much or Not Enough? specifically of one or more essential amino acids $[20,25$, 26].

Two different experimental approaches have been used to distinguish the contribution of individual or combinations of amino acids to DR benefits. In the first, amino acids are removed and replaced with sucrose or the nonessential amino acid glycine for comparison between two otherwise isocaloric diets. Using this approach, a number of different individual essential amino acids have been shown to confer DR benefits, including tryptophan, leucine, and methionine [20]. One drawback to this approach in rodents is the voluntary reduction in food intake commonly associated with such incomplete or unbalanced diets, which makes it difficult to separate the effects of specific amino acid depletion from those of overall calorie restriction. Future studies are thus required to fully disentangle the effects of amino acid restriction from overall calorie restriction, as well as to rigorously test the relative contributions of each of the 10 essential amino acids, alone or in combination, to the benefits of DR.

A second, complementary approach to assess the role of amino acids in DR benefits independent of their caloric value is to start on a restricted background (e.g., $50 \%$ DR) and add back individual or combinations of amino acids. Using this approach, increased longevity and reduced fecundity were shown to be responsive to essential amino acid re-addition in fruit flies, with a particular role for the sulfur-containing essential amino acid, methionine, and downstream effects on insulin signaling [27]. Interestingly, in the context of DR-mediated preconditioning against hepatic IRI in mice, readdition of Met and/or Cys also abrogates benefits in part through reduction of endogenous hydrogen sulfide $\left(\mathrm{H}_{2} \mathrm{~S}\right)$ generation [26]. Whether or not individual amino acids contribute through a common mechanism or independently through amino acid-specific mechanisms remains to be worked out, although examples of both will be provided in the molecular effector mechanisms section below.

It is also important to note that different organs can have different requirements for protection even against the same injury. For example, while in the renal IRI model both protein and calorie restriction contribute additively to organ protection [6], protein restriction alone contributes disproportionately to organ protection against hepatic IRI [23]. Much future work is thus required in order to determine the optimal balance of calories from protein versus sugar and fat, as well as the total calorie intake, for optimal stress resistance, which itself will likely depend on the specific surgery and its associated patient-specific risk factors. 
Nutrient-/Energy-Sensing Mechanisms Implicated in Dietary Preconditioning

The ability to sense and adjust to fluctuations in environmental nutrient/energy levels is requisite for life. In mammals, nutrient/energy sensing and neuroendocrine signaling are intricately linked in order to rapidly engage anabolism and storage in times of food excess, or mobilization and catabolism in times of scarcity. In the context of DR, it is thus not surprising that nutrient/energy sensing and hormonal pathways have been implicated in pleiotropic DR benefits [28]. While modulation of key hormonal pathways including growth hormone, insulin/ insulin-like growth factors (IGFs), and the fasting hormone FGF21 have all been associated with longevity extension by DR in rodents [29], we will focus on the amino acid and energy-sensing molecules general control nonderepressible 2 (GCN2), mechanistic target of rapamycin complex 1 (mTORC1), AMP-activated kinase (AMPK), and sirtuin-1 (SIRT1) because of their proven involvement specifically in experimental models of surgical stress resistance (Fig. 1b).

\section{GCN2}

Dietary deficiency in protein-coding amino acids is intricately linked to modulation of protein translation and stress responses through the GCN2 serine/threonine protein kinase. Typically upon dietary protein starvation or consumption of an imbalanced protein source (e.g., a monodiet consisting of rice is relatively deficient in the essential amino acid lysine), low levels of one or more amino acids promote accumulation of uncharged cognate tRNAs. Uncharged tRNAs directly activate the kinase domain of GCN2, facilitating phosphorylation of the eukaryotic translation initiation factor- $\alpha$ (eIF2 $\alpha)$. While this reduces general translation initiation efficiency, it promotes translation of specific transcripts including activating transcription factor 4 (ATF4), and increased expression of target genes involved in nonessential amino acid biosynthesis, amino acid transport, and other stress responses [25]. This cascade of events is described alternately as the amino acid starvation response (specifically when GCN2 phosphorylates eIF2 $\alpha$ ) or more generally as the integrated stress response when any of the four eIF $2 \alpha$ kinases are activated upon different stresses (e.g., PERK/ ER stress, HRI/iron stress, PKR/viral infection). Interestingly, elevated ATF4 levels have also been described in liver and isolated fibroblasts of long-lived, stress-resistant rodent models including hypopituitary Snell dwarf mice [30], and are associated with extended longevity upon nu- trient deprivation, altered ribosomal function, or rapamycin treatment in yeast [31].

In the context of dietary preconditioning against IRI, GCN2 is required for protection from renal and hepatic IRI induced by dietary tryptophan deficiency [20]. However, GCN2 does not appear to be required for preconditioning by total protein deprivation $[6,23]$, likely due to redundancy in protein-sensing mechanisms including mTORC1 as described below. GCN2 can also be activated pharmacologically by the proline tRNA synthase inhibitor halofuginone [32], originally described as an antimalarial agent and currently used in humans against scleroderma and in a clinical trial in patients with progressive advanced solid tumors (NCT00027677) [33]. In mice, pretreatment with halofuginone protects against renal IRI in a GCN2-dependent manner [20]. Nonetheless, despite providing proof-of-principle of GCN2 activation as a target for preconditioning against renal injury, the narrow window of efficacy for halofuginone due to on-target toxicity make it a less plausible strategy than DR itself.

\section{mTORC1}

While GCN2 can detect the absence of any amino acid, the presence of specific amino acids such as leucine is sensed by the protein complex mTORC1, a serine/threonine kinase that integrates information on amino acid, energy, and growth factor availability in order to effect cell fate decisions regarding anabolism versus catabolism $[34,35]$. Amino acids are sensed by mTORC1 via an upstream mechanism involving the Rag family GTPases and a regulatory complex referred to as the Ragulator [36] that anchors MTORC1 at the lysosomal surface.

Hepatic mTORC1 activity is reduced in mice preconditioned on a protein-free diet for 1 week, coinciding with protection against hepatic IRI [23]. Whether this is a direct result of reduced amino acid (leucine) levels, or more likely a reduction of growth factor levels also required for mTORC 1 activation, remains to be determined. However consistent with the importance of reduced mTORC1 in dietary preconditioning, liver-specific deletion of the upstream mTORC1 repressor gene TSC1, results in constitutive hepatic mTORC1 activation independent of diet, and prevents protein restriction from preconditioning against hepatic IRI [23]. Interestingly, preconditioning by tryptophan restriction alone does not require the TSC complex, suggesting that different mechanisms might be mediating the effects of individual amino acid and total protein-deficient diets, potentially via parallel action of GCN2 and mTORC1 pathways [23]. 
Rapamycin (Sirolimus) is a partial mTORC1 inhibitor used in humans to prevent $\mathrm{T}$ and $\mathrm{B}$ cell-mediated organ transplant rejection, and also as a vascular stent coating to prevent intimal hyperplasia [37]. In mice, long-term rapamycin treatment extends longevity $[38,39]$, but does not protect mice from hepatic IRI despite reducing hepatic mTORC1 signaling [23]. Thus, in the context of dietary preconditioning, reduced $\mathrm{mTORC} 1$ signaling may be necessary but insufficient for protection. Alternately, side effects of rapamycin including reduced insulin sensitivity may have a larger negative impact in the context of hepatic IRI than in longevity extension [38]. Thus, despite having identified 2 amino acid-sensing pathways targetable with pharmacological agents, much work remains to be done in order to understand how to use such agents to successfully mimic the beneficial effects of dietary preconditioning.

\section{$A M P K$}

AMPK is a conserved energy-sensing protein kinase present in all eukaryotes as heterotrimers comprising catalytic $\alpha$-subunit and regulatory $\beta$ - and $\gamma$-subunits. AMPK is allosterically activated when cellular energy is low via AMP and/or ADP as well as by several upstream kinases. Activation of AMPK promotes energy production by increasing glucose uptake in the short term and facilitating a switch to oxidative metabolism in the long term. It also reduces anabolic mTORC1 signaling by phosphorylating the mTORC1 inhibitor, TSC2. AMPK is activated upon fasting or DR regimens involving enforced food restriction. Somewhat surprisingly, it is also activated upon protein restriction independent of calorie intake [6]. Like mTORC1 signaling with which AMPK is intricately intertwined, this could reflect a change in the growth factor environment induced by protein restriction, resulting in a metabolic state resembling energy deficiency. The essential nature of AMPK function and redundancy in catalytic AMPK subunit genes complicates testing the genetic requirement for AMPK in dietary preconditioning. However, AMPK activation by the allosteric activator 5 -aminoimidazole-4-carboxamide ribonucleotide (AICAR) protects against ischemic injury in rats when given at high doses before injury [40]. AMPK activation by the endogenous adipokine adiponectin, which is increased upon DR, is also essential for the cardioprotective effects of DR against MI [41].

\section{SIRT1}

Under conditions of nutritional and environmental stress (fasting, DR, and exercise), the NAD+-dependent deacetylase SIRT1 stimulates mitochondrial biogenesis, energy homeostasis and cell survival by regulating the acetylation and activity of transcription factors, including the peroxisome roliferator-activated receptor- $\gamma$ coactivator-1 $\alpha$ (PGC-1 $\alpha)$ and forkhead box O (FOXO) $[42,43]$. In the context of surgical stress, SIRT1 activation protects in mouse models of hepatic IRI and stroke (middle cerebral artery occlusion), while genetic ablation increases sensitivity to these injuries $[44,45]$. In addition, 3 months of $40 \% \mathrm{DR}$ preconditioning protects in an ex vivo model of myocardial IRI via an eNOS-dependent increase in SIRT1 [46]. Sirtuins have attracted considerable attention as drug targets and are discussed in detail elsewhere [47, 48]. Of interest, hepatocytes treated with either the sirtuin activators resveratrol or SRT1720 were protected in an in vitro model of IRI [49]. In vivo, treatment of mice with sirtuin activator 3 reduced infarct volume following middle cerebral artery occlusion [44].

\section{Molecular Effector Mechanisms Underlying Dietary Preconditioning}

Because of the plethora of physiological and molecular changes that occur even upon short-term restriction of a single essential amino acid from the diet, identification of critical downstream mechanisms of DR-mediated protection against surgical stress is challenging. Elucidation of upstream nutrient-sensing pathways such as GCN2 and mTORC1, for which genetic full-body or tissue-specific knockout models are available, has proven a critical step forward. Using experimental designs in which dietary interventions are combined with genetic models lacking upstream nutrient sensors that fail to gain protection upon DR, two major downstream mechanisms involving increased prosurvival insulin signaling and endogenous $\mathrm{H}_{2} \mathrm{~S}$ production have recently been elucidated.

\section{Increased Prosurvival Insulin Signaling}

Improved insulin sensitivity is one of the metabolic hallmarks common to extended longevity models including interventions such as DR, and genetic models of reduced signaling through the growth hormone/insulin/ IGF/mTORC1 axis [28]. DR improves insulin sensitivity in part through lowering circulating insulin levels. This in turn reduces mTORC1 activity and its associated feedback inhibition of insulin receptor signaling, facilitating improved insulin sensitivity [50].

Mice lacking TSC1 specifically in hepatocytes (LiTsc1KO) display constitutive hepatic mTORC1 activity and profound hepatic insulin resistance due to feed- 
back inhibition of insulin receptor signaling, even upon DR despite low circulating insulin levels [23]. In the hepatic IRI model, LTsc1KO mice fail to gain the benefit of dietary preconditioning in part due to insulin resistance. Consistent with this, specific inhibition of insulin signaling via genetic deletion of the insulin receptor in hepatocytes, or via pharmacological inhibition with the PI3K inhibitor wortmannin in wild-type mice, significantly impinges on the ability of short-term DR to protect against hepatic IRI [23].

How does the DR-mediated improvement in hepatic insulin sensitivity contribute to protection from hepatic IRI? In addition to regulating energy metabolism, insulin can act as a prosurvival factor via negative regulation of apoptosis. Consistent with this mechanism of action, circulating insulin levels and antiapoptotic signaling are both increased in the hours after liver reperfusion in wildtype mice preconditioned on DR, while this effect is absent in mice with constitutive insulin resistance [23]. Taken together, these data suggest that a major mechanism of DR action is via increased insulin sensitivity prior to an injury, which then facilitates increased prosurvival signaling and reduced hepatocyte apoptosis after injury. Interestingly, GCN2 activation upon amino acid restriction also improves insulin sensitivity [51], and this correlates with increased resistance to IRI [20].

It is important to note that as with insulin, reduced circulating IGF-1 is characteristic of numerous genetic and dietary models of extended longevity [52]. In rodents, short-term fasting reduces IGF-1 and protects against chemotherapeutic toxicity [53]. Protein/amino acid restriction also decreases IGF- 1 in rodents, as it does in humans [54] in a GCN2-dependent manner [20]; however, whether or not this plays a role in dietary preconditioning against surgical stress remains untested.

Benefits of reduced IGF-1 are thought to work through activation of stress resistance pathways normally suppressed by insulin/IGF-1 signaling, for example expression of antioxidant-encoding genes [53]. However, increased IGF-1 is also associated in many contexts with increased stress resistance and improved outcome after IRI [55]. How can both increased and decreased IGF-1 signaling be beneficial? In the context of acute stress resistance, as long as both of these mechanisms are separated in time relative to the acute stress, they are not necessarily mutually exclusive: reduced IGF- 1 signaling during the dietary preconditioning period may increase FOXO-dependent antioxidant gene expression, while increased antiapoptotic IGF-1 signaling after injury may prevent cell death as described above for insulin.

\section{Increased Endogenous $\mathrm{H}_{2} \mathrm{~S}$}

$\mathrm{H}_{2} \mathrm{~S}$ is a gas easily identified by its distinctive odor of rotten eggs. Although toxic at high levels, endogenously produced $\mathrm{H}_{2} \mathrm{~S}$ by one of three evolutionarily conserved enzymes is now recognized to have pleiotropic cytoprotective, anti-inflammatory and vasodilatory effects resulting in cardioprotection and resistance to ischemic injury $[26,56]$. In the context of dietary preconditioning against hepatic IRI, mice lacking the $\mathrm{H}_{2} \mathrm{~S}$-producing enzyme cystathionine $\gamma$-lyase (CGL) fail to gain the protective effects of DR. Furthermore, adenoviral overexpression of CGL in the liver, or delivery of $\mathrm{H}_{2} \mathrm{~S}$ itself, recapitulate DR-like benefits without the need for any dietary intervention [26]. These findings establish the importance of endogenously produced $\mathrm{H}_{2} \mathrm{~S}$ in DR-mediated protection from hepatic IRI.

$\mathrm{H}_{2} \mathrm{~S}$ has numerous potential mechanisms of action, including sulfhydration, or formation of -SSH moieties on surface-exposed Cys residues, of an ever-growing list of protein targets. In endothelial cells, for example, such targets include the Kir6.2 regulatory subunit of the $\mathrm{K}_{\mathrm{ATP}}$ channel and the VEGF receptor leading to vessel relaxation and angiogenesis, respectively $[57,58] . \mathrm{H}_{2} \mathrm{~S}$ also has direct antioxidant properties, and can participate in mitochondrial energy production by donating electrons to the mitochondrial electron transport chain protein $S Q R$, with a potential role in protection from ischemia. Since pharmacological delivery of $\mathrm{H}_{2} \mathrm{~S}$ also protects in models of surgical stress, as well as more broadly in preclinical models of cardiovascular disease [26], it remains to be seen if supplementation with exogenous sources of $\mathrm{H}_{2} \mathrm{~S}$, or increased endogenous $\mathrm{H}_{2} \mathrm{~S}$ production through dietary or other means, will ultimately turn out to be more beneficial in the context of surgical stress resistance.

\section{Implications for Clinical Translation in Surgery}

The findings that short-term fasting or restriction of food intake - on the order of days to a week - leads to robust functional benefits in rodents has profound implications for the mechanism of DR action in mammals. Rather than previous notions of DR as an intervention whose benefits accumulate over long periods of time due to reduced calorie intake, DR is now viewed as a rapid adaptation to the mild stress of calorie and/or nutrient deprivation with the potential to protect against many other forms of stress. This new understanding has important practical implications for attempts to leverage DR against clinically relevant endpoints, including planned surgery. 
If future clinical trials identify brief DR regimens or pharmacological DR mimetics that are safe and effective against the stress and potential complications of surgery, how would this change current preoperative nutritional standards? With few exceptions such as bariatric surgery discussed below, there is currently no consensus on what should or should not be eaten up to 1 day prior to surgery, so long as the patient is not suffering from malnutrition. Starting from the day before surgery, optimization of nutrition is a cornerstone (along with preoperative counseling, standardized analgesic and anesthetic regimens, and early mobilization after surgery) of enhanced recovery after surgery (ERAS) protocols for perioperative care designed to achieve early recovery after surgical procedures $[59,60]$. ERAS nutritional optimization calls for preoperative oral carbohydrate loading, including $100 \mathrm{~g}$ of carbohydrate administered the day before and $50 \mathrm{~g}$ the day of the surgery, and is designed to alleviate surgery-induced insulin resistance and reduce patient discomfort in the form of hunger and thirst $[59,60]$.

A concept that is embedded in ERAS nutritional guidelines - namely that a catabolic state prior to surgery is undesirable, or even detrimental - has also influenced the long-standing "NPO after midnight" recommendation designed to prevent bronchoaspiration during anesthesia. Current guidelines have shortened the fasting period to $8 \mathrm{~h}$ after a meal containing fat or meat, and to $2 \mathrm{~h}$ after ingestion of clear fluids including beverages designed for carbohydrate loading [61]. Based on these current practices, extending the fasting regimen, or simply reducing protein/calorie intake, for a period of days prior to surgery specifically to leverage benefits associated with DR would thus represent a paradigm shift in perioperative nutrition.

Although long-term DR has been popularized for its ability to extend longevity, it is still largely unknown in the clinical literature and often confused with starvation. Furthermore, long-term DR has been associated with impaired wound healing and immunosuppression in animal models, and thus suspected of potentially increasing risk of bacterial infection $[16,62]$. Nonetheless, a form of preoperative DR is already the standard of care in one surgical intervention, albeit for a different reason. In the context of bariatric surgery, current preoperative guidelines call for low- or very-low-calorie diets (although typically with protein supplementation) to reduce liver mass and facilitate the surgical procedure [63]. Importantly, studies specifically designed to test the potential efficacy of shortterm DR in clinical applications, including living organ donor surgery $[64,65]$ and chemotherapy treatment $[66$, 67], have demonstrated safety and feasibility in these patient populations. Thus, while short-term DR/fasting appears feasible and safe in select populations, efficacy in mitigating risk more broadly in elective surgery, and in particular in high-risk individuals and procedures, remains to be rigorously tested.

\section{Conclusions}

Long-term DR is a well-established interventional method of life span and health span extension across species under defined experimental conditions, with some metabolic health benefits in humans confirmed in prospective clinical trials $[3,4,68]$. Short-term DR or fasting prior to surgery preconditions against a variety of complications in preclinical models $[6,8,10,20,23,26]$. Currently, the duration of preoperative fasting used as an "anesthetic precaution" in humans is likely too short to tap into DR benefits, while the progressive clinical application of ERAS nutritional guidelines promotes an alternate although not mutually exclusive concept of increased nutrition immediately prior to surgery. Future clinical trials are required to test the safety, feasibility, and potential efficacy of short-term DR, including extended periods of fasting, to reduce risk of surgical complications and improve outcomes. If successful, this approach has the potential to change the paradigm for preoperative nutritional care based on concepts derived from research into the basic biology of aging.

\section{References}

Is Overnight Fasting before Surgery Too Much or Not Enough?
1 Speakman JR, Mitchell SE: Caloric restriction. Mol Aspects Med 2011;32:159-221.

2 McCay CM, Crowell MF, Maynard LA: The effect of retarded growth upon the length of life span and upon the ultimate body size. 1935. Nutrition 1989;5:155-171; discussion 172.

3 Ravussin E, Redman LM, Rochon J, Das SK, Fontana L, Kraus WE, Romashkan S, Wil- liamson DA, Meydani SN, Villareal DT, Smith SR, Stein RI, Scott TM, Stewart TM, Saltzman E, Klein S, Bhapkar M, Martin CK, Gilhooly CH, Holloszy JO, Hadley EC, Roberts SB; CALERIE Study Group: A 2-year randomized controlled trial of human caloric restriction: feasibility and effects on predictors of health span and longevity. J Gerontol A Biol Sci Med Sci 2015;70:1097-1104. 
4 Fontana L, Klein S: Aging, adiposity, and calorie restriction. JAMA 2007;297:986-994.

5 Mitchell JR, Beckman JA, Nguyen LL, Ozaki CK: Reducing elective vascular surgery perioperative risk with brief preoperative dietary restriction. Surgery 2013;153:594-598.

6 Robertson LT, Treviño-Villarreal JH, Mejia P, Grondin Y, Harputlugil E, Hine C, Vargas D, Zheng H, Ozaki CK, Kristal BS, Simpson SJ, Mitchell JR: Protein and calorie restriction contribute additively to protection from renal ischemia reperfusion injury partly via leptin reduction in male mice. J Nutr 2015;145: 1717-1727.

7 Mauro CR, Tao M, Yu P, Treviño-Villerreal $\mathrm{JH}$, Longchamp A, Kristal BS, Ozaki CK, Mitchell JR: Preoperative dietary restriction reduces intimal hyperplasia and protects from ischemia-reperfusion injury. J Vasc Surg 2016;63:500-509.e1.

8 Varendi K, Airavaara M, Anttila J, Vose S, Planken A, Saarma M, Mitchell JR, Andressoo JO: Short-term preoperative dietary restriction is neuroprotective in a rat focal stroke model. PLoS One 2014;9:e93911.

9 Verweij M, van Ginhoven TM, Mitchell JR, Sluiter W, van den Engel S, Roest HP, Torabi E, Ijzermans JN, Hoeijmakers JH, de Bruin RW: Preoperative fasting protects mice against hepatic ischemia/reperfusion injury: mechanisms and effects on liver regeneration. Liver Transpl 2011;17:695-704.

10 Mitchell JR, Verweij M, Brand K, van de Ven M, Goemaere N, van den Engel S, Chu T, Forrer F, Müller C, de Jong M, van IJcken W, IJzermans JN, Hoeijmakers JH, de Bruin RW: Short-term dietary restriction and fasting precondition against ischemia reperfusion injury in mice. Aging Cell 2010;9:40-53.

11 Desborough JP: The stress response to trauma and surgery. Br J Anaesth 2000;85:109-117.

12 Fleisher LA, Fleischmann KE, Auerbach AD, Barnason SA, Beckman JA, Bozkurt B, Davila-Roman VG, Gerhard-Herman MD, Holly TA, Kane GC, Marine JE, Nelson MT, Spencer CC, Thompson A, Ting HH, Uretsky BF, Wijeysundera DN: 2014 ACC/AHA guideline on perioperative cardiovascular evaluation and management of patients undergoing noncardiac surgery: a report of the American College of Cardiology/American Heart Association Task Force on Practice Guidelines. Circulation 2014;130:e278-e333.

13 Vinten-Johansen J, Nakanishi K: Postcardioplegia acute cardiac dysfunction and reperfusion injury. J Cardiothorac Vasc Anesth 1993; $7: 6-18$.

14 Chambers DJ, Fallouh HB: Cardioplegia and cardiac surgery: pharmacological arrest and cardioprotection during global ischemia and reperfusion. Pharmacol Ther 2010;127:4152.

15 Wald R, Waikar SS, Liangos O, Pereira BJ, Chertow GM, Jaber BL: Acute renal failure after endovascular vs open repair of abdominal aortic aneurysm. J Vasc Surg 2006;43:460466; discussion 466.
16 Chandrasekar B, Nelson JF, Colston JT, Freeman GL: Calorie restriction attenuates inflammatory responses to myocardial ischemia-reperfusion injury. Am J Physiol Heart Circ Physiol 2001;280:H2094-H2102.

$17 \mathrm{Yu}$ ZF, Mattson MP: Dietary restriction and 2-deoxyglucose administration reduce focal ischemic brain damage and improve behavioral outcome: evidence for a preconditioning mechanism. J Neurosci Res 1999;57:830-839.

18 Robertson LT, Mitchell JR: Benefits of shortterm dietary restriction in mammals. Exp Gerontol 2013;48:1043-1048.

19 Sumimoto R, Southard JH, Belzer FO: Livers from fasted rats acquire resistance to warm and cold ischemia injury. Transplantation 1993;55:728-732.

20 Peng W, Robertson L, Gallinetti J, Mejia P, Vose S, Charlip A, Chu T, Mitchell JR: Surgical stress resistance induced by single amino acid deprivation requires Gcn2 in mice. Sci Transl Med 2012;4:118ra111.

21 Plunet WT, Streijger F, Lam CK, Lee JH, Liu J, Tetzlaff W: Dietary restriction started after spinal cord injury improves functional recovery. Exp Neurol 2008;213:28-35.

22 Weindruch R, Walford RL, Fligiel S, Guthrie D: The retardation of aging in mice by dietary restriction: longevity, cancer, immunity and lifetime energy intake. J Nutr 1986;116:641-654.

23 Harputlugil E, Hine C, Vargas D, Robertson L, Manning BD, Mitchell JR: The TSC complex is required for the benefits of dietary protein restriction on stress resistance in vivo. Cell Rep 2014;8:1160-1170.

24 Speakman JR, Mitchell SE, Mazidi M: Calories or protein? The effect of dietary restriction on lifespan in rodents is explained by calories alone. Exp Gerontol, Epub ahead of print.

25 Gallinetti J, Harputlugil E, Mitchell JR: Amino acid sensing in dietary-restriction-mediated longevity: roles of signal-transducing kinases GCN2 and TOR. Biochem J 2013;449: $1-10$

26 Hine C, Harputlugil E, Zhang Y, Ruckenstuhl C, Lee BC, Brace L, Longchamp A, TreviñoVillarreal JH, Mejia P, Ozaki CK, Wang R, Gladyshev VN, Madeo F, Mair WB, Mitchell JR: Endogenous hydrogen sulfide production is essential for dietary restriction benefits. Cell 2015;160:132-144.

27 Grandison RC, Piper MD, Partridge L: Amino-acid imbalance explains extension of lifespan by dietary restriction in Drosophila. Nature 2009;462:1061-1064.

28 Fontana L, Partridge L, Longo VD: Extending healthy life span - from yeast to humans. Science 2010;328:321-326.

29 Longo VD, Antebi A, Bartke A, Barzilai N, Brown-Borg HM, Caruso C, Curiel TJ, de Cabo R, Franceschi C, Gems D, Ingram DK, Johnson TE, Kennedy BK, Kenyon C, Klein S, Kopchick JJ, Lepperdinger G, Madeo F, Mirisola MG, Mitchell JR, Passarino G, Rudolph KL, Sedivy JM, Shadel GS, Sinclair DA, Spindler SR, Suh Y, Vijg J, Vinciguerra M, Fontana
L: Interventions to slow aging in humans: are we ready? Aging Cell 2015;14:497-510.

$30 \mathrm{Li}$ W, Miller RA: Elevated ATF4 function in fibroblasts and liver of slow-aging mutant mice. J Gerontol A Biol Sci Med Sci 2015;70: 263-272.

31 Kubota $\mathrm{H}$, Obata $\mathrm{T}$, Ota K, Sasaki T, Ito T: Rapamycin-induced translational derepression of GCN4 mRNA involves a novel mechanism for activation of the eIF2 alpha kinase GCN2. J Biol Chem 2003;278:20457-20460.

32 Sundrud MS, Koralov SB, Feuerer M, Calado DP, Kozhaya AE, Rhule-Smith A, Lefebvre RE, Unutmaz D, Mazitschek R, Waldner $\mathrm{H}$, Whitman M, Keller T, Rao A: Halofuginone inhibits TH17 cell differentiation by activating the amino acid starvation response. Science 2009;324:1334-1338.

33 Pines M: Halofuginone for fibrosis, regeneration and cancer in the gastrointestinal tract. World J Gastroenterol 2014;20:14778-14786.

34 Jewell JL, Russell RC, Guan KL: Amino acid signalling upstream of mTOR. Nat Rev Mol Cell Biol 2013;14:133-139.

35 Dibble CC, Manning BD: Signal integration by mTORC1 coordinates nutrient input with biosynthetic output. Nat Cell Biol 2013;15: 555-564.

36 Efeyan A, Comb WC, Sabatini DM: Nutrientsensing mechanisms and pathways. Nature 2015;517:302-310.

37 Waldner M, Fantus D, Solari M, Thomson AW: New perspectives on mTOR inhibitors (rapamycin, rapalogs and TORKinibs) in transplantation. Br J Clin Pharmacol 2016;82: 1158-1170.

38 Lamming DW, Ye L, Katajisto P, Goncalves MD, Saitoh M, Stevens DM, Davis JG, Salmon $\mathrm{AB}$, Richardson A, Ahima RS, Guertin DA, Sabatini DM, Baur JA: Rapamycin-induced insulin resistance is mediated by mTORC2 loss and uncoupled from longevity. Science 2012;335:1638-1643.

39 Harrison DE, Strong R, Sharp ZD, Nelson JF, Astle CM, Flurkey K, Nadon NL, Wilkinson JE, Frenkel K, Carter CS, Pahor M, Javors MA, Fernandez E, Miller RA: Rapamycin fed late in life extends lifespan in genetically heterogeneous mice. Nature 2009;460:392-395.

40 Lempiäinen J, Finckenberg P, Levijoki J, Mervaala E: AMPK activator AICAR ameliorates ischaemia reperfusion injury in the rat kidney. Br J Pharmacol 2012;166:1905-1915.

41 Shinmura K, Tamaki K, Saito K, Nakano Y, Tobe T, Bolli R: Cardioprotective effects of short-term caloric restriction are mediated by adiponectin via activation of AMP-activated protein kinase. Circulation 2007;116:28092817.

42 Daitoku H, Sakamaki J, Fukamizu A: Regulation of FoxO transcription factors by acetylation and protein-protein interactions. Biochim Biophys Acta 2011;1813:1954-1960.

43 Cantó C, Auwerx J: PGC-1alpha, SIRT1 and AMPK, an energy sensing network that controls energy expenditure. Curr Opin Lipidol 2009;20:98-105. 
44 Hernández-Jiménez $\mathrm{M}$, Hurtado $\mathrm{O}$, Cuartero MI, Ballesteros I, Moraga A, Pradillo JM, McBurney MW, Lizasoain I, Moro MA: Silent information regulator 1 protects the brain against cerebral ischemic damage. Stroke 2013;44:2333-2337.

45 Yang Y, Zhang S, Fan C, Yi W, Jiang S, Di S, Ma Z, Hu W, Deng C, Lv J, Li T, Nie Y, Jin Z: Protective role of silent information regulator 1 against hepatic ischemia: effects on oxidative stress injury, inflammatory response, and MAPKs. Expert Opin Ther Targets 2016;20: 519-531.

46 Yamamoto T, Tamaki K, Shirakawa K, Ito K, Yan X, Katsumata Y, Anzai A, Matsuhashi T, Endo J, Inaba T, Tsubota K, Sano M, Fukuda K, Shinmura K: Cardiac Sirt1 mediates the cardioprotective effect of caloric restriction by suppressing local complement system activation after ischemia-reperfusion. Am J Physiol Heart Circ Physiol 2016;310:H1003H1014.

47 Hubbard BP, Sinclair DA: Small molecule SIRT1 activators for the treatment of aging and age-related diseases. Trends Pharmacol Sci 2014;35:146-154.

48 Sinclair DA, Guarente L: Small-molecule allosteric activators of sirtuins. Annu Rev Pharmacol Toxicol 2014;54:363-380.

49 Biel TG, Lee S, Flores-Toro JA, Dean JW, Go KL, Lee MH, Law BK, Law ME, Dunn WA, Zendejas I, Behrns KE, Kim JS: Sirtuin 1 suppresses mitochondrial dysfunction of ischemic mouse livers in a mitofusin 2-dependent manner. Cell Death Differ 2016;23:279-290.

50 Howell JJ, Manning BD: mTOR couples cellular nutrient sensing to organismal metabolic homeostasis. Trends Endocrinol Metab 2011;22:94-102.

51 Xiao F, Huang Z, Li H, Yu J, Wang C, Chen S, Meng Q, Cheng Y, Gao X, Li J, Liu Y, Guo F: Leucine deprivation increases hepatic insulin sensitivity via GCN2/mTOR/S6K1 and AMPK pathways. Diabetes 2011;60:746-756.

52 Russell SJ, Kahn CR: Endocrine regulation of ageing. Nat Rev Mol Cell Biol 2007;8:681691.

53 Lee C, Safdie FM, Raffaghello L, Wei M, Madia F, Parrella E, Hwang D, Cohen P, Bianchi G, Longo VD: Reduced levels of IGF-I mediate differential protection of normal and can- cer cells in response to fasting and improve chemotherapeutic index. Cancer Res 2010;70: 1564-1572.

54 Fontana L, Weiss EP, Villareal DT, Klein S, Holloszy JO: Long-term effects of calorie or protein restriction on serum IGF-1 and IGFBP-3 concentration in humans. Aging Cell 2008;7:681-687.

55 Ungvari Z, Csiszar A: The emerging role of IGF-1 deficiency in cardiovascular aging: recent advances. J Gerontol A Biol Sci Med Sci 2012;67:599-610.

56 Kondo K, Bhushan S, King AL, Prabhu SD, Hamid T, Koenig S, Murohara T, Predmore BL, Gojon G, Wang R, Karusula N, Nicholson CK, Calvert JW, Lefer DJ: $\mathrm{H}_{2}$ S protects against pressure overload-induced heart failure via upregulation of endothelial nitric oxide synthase. Circulation 2013;127:1116-1127.

57 Mustafa AK, Gadalla MM, Sen N, Kim S, Mu W, Gazi SK, Barrow RK, Yang G, Wang R, Snyder SH: H2S signals through protein Ssulfhydration. Sci Signal 2009;2:ra72.

58 Tao BB, Liu SY, Zhang CC, Fu W, Cai WJ, Wang Y, Shen Q, Wang MJ, Chen Y, Zhang LJ, Zhu YZ, Zhu YC: VEGFR2 functions as an $\mathrm{H} 2 \mathrm{~S}$-targeting receptor protein kinase with its novel Cys1045-Cys1024 disulfide bond serving as a specific molecular switch for hydrogen sulfide actions in vascular endothelial cells. Antioxid Redox Signal 2013;19:448464.

59 Coolsen MM, Wong-Lun-Hing EM, van Dam RM, van der Wilt AA, Slim K, Lassen K, Dejong $\mathrm{CH}$ : A systematic review of outcomes in patients undergoing liver surgery in an enhanced recovery after surgery pathways. HPB (Oxford) 2013;15:245-251

60 Gustafsson UO, Scott MJ, Schwenk W, Demartines N, Roulin D, Francis N, McNaught CE, Macfie J, Liberman AS, Soop M, Hill A, Kennedy RH, Lobo DN, Fearon K, Ljungqvist O; Enhanced Recovery After Surgery (ERAS) Society, for Perioperative Care; European Society for Clinical Nutrition and Metabolism (ESPEN); International Association for Surgical Metabolism and Nutrition (IASMEN): Guidelines for perioperative care in elective colonic surgery: Enhanced Recovery After Surgery (ERAS ${ }^{\circledR}$ ) Society recommendations. World J Surg 2013;37:259-284.
61 American Society of Anesthesiologists Committee on Standards and Practice Parameters: Practice guidelines for preoperative fasting and the use of pharmacologic agents to reduce the risk of pulmonary aspiration: application to healthy patients undergoing elective procedures: an updated report by the American Society of Anesthesiologists Committee on Standards and Practice Parameters. Anesthesiology 2011;114:495-511.

62 Hunt ND, Li GD, Zhu M, Miller M, Levette A, Chachich ME, Spangler EL, Allard JS, Hyun DH, Ingram DK, de Cabo R: Effect of calorie restriction and refeeding on skin wound healing in the rat. Age (Dordr) 2012;34:14531458.

63 Van Nieuwenhove Y, Dambrauskas Z, Campillo-Soto A, van Dielen F, Wiezer R, Janssen I, Kramer M, Thorell A: Preoperative very low-calorie diet and operative outcome after laparoscopic gastric bypass: a randomized multicenter study. Arch Surg 2011;146: 1300-1305.

64 Jongbloed F, de Bruin RW, Klaassen RA, Beekhof P, van Steeg H, Dor FJ, van der Harst E, Dollé ME, IJzermans JN: Short-term preoperative calorie and protein restriction is feasible in healthy kidney donors and morbidly obese patients scheduled for surgery. Nutrients 2016;8:E306.

65 van Ginhoven TM, de Bruin RW, Timmermans M, Mitchell JR, Hoeijmakers JH, Ijzermans JN: Pre-operative dietary restriction is feasible in live-kidney donors. Clin Transplant 2011;25:486-494.

66 de Groot S, Vreeswijk MP, Welters MJ, Gravesteijn G, Boei JJ, Jochems A, Houtsma D, Putter H, van der Hoeven JJ, Nortier JW, Pijl $\mathrm{H}$, Kroep JR: The effects of short-term fasting on tolerance to (neo) adjuvant chemotherapy in HER2-negative breast cancer patients: a randomized pilot study. BMC Cancer 2015; 15:652.

67 Safdie FM, Dorff T, Quinn D, Fontana L, Wei $\mathrm{M}$, Lee C, Cohen P, Longo VD: Fasting and cancer treatment in humans: a case series report. Aging (Albany NY) 2009;1:988-1007.

68 Redman LM, Ravussin E: Endocrine alterations in response to calorie restriction in humans. Mol Cell Endocrinol 2009;299:129136 